\title{
Aktuelle Herausforderungen für das Preiscontrolling
}

\author{
Transfer
}

Anderson, E. T., Simester, D. I. (2004), Long-Run Effects of Promotion Depth on New Versus Established Customers: Three Field Studies, in: Marketing Science, 23, 1, 4- 20.

Gronholdt, L., Martensen, A., Kristensen, K. (2000), The Relationship between Customer Satisfaction and Loyality: Cross-Industry Differences, in: Total Quality Management, 11, 4/5/6, $509-514$

Hermann, A., Xia, L., Monroe, K. B., Huber, K. (2007), The Influence of Price Fairness on Customer Satisfaction: An Empirical Test in the Context of Automobile Purchases, in: Journal of Product \& Brand Management, 16, 1, $49-58$.

Joseph, K. (2001), On the Optimality of Delegating Pricing Authority to the Sales Force, in: Journal of Marketing, 65, 1, $62-70$.

Mishra, B. K., Prasad, A. (2004), Centralized Pricing Versus Delegating Pricing to the Salesforce Under Information Asymmetry, in: Marketing Science, 23, 1, $21-27$.

Sun, B. (2005), Promotion Effect on Endogenous Consumption, in: Marketing Science, 24, 3, $430-443$.

Betrachtet man die wissenschaftlichen Veröffentlichungen zum Preiscontrolling als Teilgebiet des Marketingcontrollings (vgl. Reinecke/Janz 2007) in den letzten Jahren, lassen sich zahlreiche Herausforderungen identifizieren. Drei häufig thematisierte Bereiche des Preiscontrollings umfassen dabei (1) die Steuerung bzw. Berücksichtigung der wahrgenommenen Preisfairness, (2) die Ausgestaltung von Preispromotionen sowie (3) die Delegation von Preiskompetenz an den Außendienst. Im Rahmen des Beitrags werden aktuelle Studien zu diesen Feldern zusammengefasst und deren wissenschaftliche Erkenntnisse auf die Praxis übertragen. Zudem wird aufgezeigt, dass die drei erläuterten As- pekte des Preiscontrollings nicht unabhängig voneinander, sondern in einem Gesamtzusammenhang zu sehen sind.

\section{Steuerung bzw. Berücksichtigung der wahrgenommenen Preisfairness im Preiscontrolling}

Steigende Gewinne und gleichzeitige Preiserhöhungen einzelner Unternehmen (Beispiel Deutsche Bahn bzw. E.ON) haben in Deutschland verstärkt zu öffentlichen Debatten über die ethische Korrektheit solcher Maßnahmen geführt. Auch in der Wissenschaft ist diese Herausforderung in Untersuchungen zum Thema Preisfairness in den letzten Jahren vermehrt behandelt worden. Anders als in den öffentlichen Diskussionen wird dort jedoch das Ziel verfolgt, statistisch signifikante Auswirkungen der Preisfairness auf das Konsumentenverhalten und damit den Erfolg von Unternehmen zu erforschen. Hermann/Xia/Monroe/Huber (2007) untersuchen beispielsweise in einem Strukturgleichungsmodell den Einfluss der wahrgenommenen Preisfairness auf die Kundenzufriedenheit. Zur Gewinnung der Daten wurden insgesamt 246 Personen bei ihrem Autohändler von einem Marktforschungsunternehmen zu ihrem getätigten Autokauf interviewt. Die Autoren unterscheiden in ihrer Analyse zwei Arten von wahrgenommener Preisfairness: Wahrgenommene Preisfairness des Angebotes und wahrgenommene Fairness des Preissetzungsverfahrens des Händlers. Insgesamt weist die Studie interessante Ergebnisse für die Praxis auf, aus denen sich folgende Implikationen ableiten lassen:

\section{Generelle Berücksichtigung von}

Preisfairness bei der Preisgestaltung

Die Wahrnehmung des Preises beeinflusst einerseits direkt, anderseits indirekt über die wahrgenommene Preisfairness die Zufriedenheit mit dem Kauf. Die Ergebnisse weisen auf einen sehr starken indirekten
Effekt über die wahrgenommene Preisfairness hin. Betrachtet man dieses Ergebnis im Zusammenhang mit jenen Studien, die einen signifikanten, positiven Effekt der Kundenzufriedenheit auf die Kundenloyalität und letztlich die Profitabilität nachweisen konnten (vgl. Gronholdt/Martensen/Kristensen 2000), lässt sich folgern, dass eine generelle Berücksichtigung und Steuerung von Preisfairnessaspekten bei der Preisgestaltung einen nicht zu unterschätzenden Erfolgsfaktor darstellt.

Steigerung der Preisfairness durch Berücksichtigung der psychologischen Verfassung der Konsumenten

Die Generierung eines schnellen Umsatzes infolge einer Notsituation des Kunden kann verheerende Auswirkungen auf die wahrgenommene Preisfairness und die langfristige Profitabilität haben. Die Ergebnisse zeigen, dass die wahrgenommene Preisfairness des Angebotes dann besonders niedrig ist, wenn sich der Kunde zum Kauf eines Produktes gezwungen fühlt und daher besonders ,verletzlich“ ist. Verkäufer sollten daher nicht nur vermeiden, ihre Kunden auszunutzen, sondern auch gerade in Notsituationen, in welchen das Empfinden des Ausgenutztwerdens groß ist, besonders sensibel gegenüber der psychologischen Verfassung der Käufer sein und eine faire Behandlung der Käufer sicherstellen. Dies kann zu einer Erhöhung der wahrgenommenen Preisfairness führen, ohne den Preis verändern zu müssen. Eine Berücksichtigung solcher Überlegungen stellt jedoch eine große Herausforderung für das Controlling dar.

\section{Steigerung der Preisfairness durch} Preistransparenz

Besonders bei komplexen Käufen ist es wichtig, eine hohe Preistransparenz zu schaffen und dem Kunden die Zusammensetzung des Gesamtpreises zu erklären. Dies steigert die wahrgenommene Fairness des Preissetzungsverfahrens und führt infolgedessen zu einer hohen $\mathrm{Ge}$ - 
samtzufriedenheit mit dem Kauf. Hierbei ist insbesondere bei Preiserhöhungen die Kommunikation der Ursachen wichtig. Beispielsweise wird ein höherer Preis eher vom Konsumenten akzeptiert, wenn externe, vom Anbieter nicht zu kontrollierende Gründe wie ein Anstieg der Rohstoffpreise dem Kunden aktiv kommuniziert werden. Bei Entscheidungen über die Offenlegung der Preiszusammensetzung sollte jedoch berücksichtigt werden, dass eine hohe Preistransparenz auch zu einer besseren Vergleichbarkeit von Angeboten führen kann. Dies ist insbesondere bei weniger komplexen Produkten der Fall. Bei komplexen Produkten hingegen überwiegen die Vorteile einer hohen Preistransparenz insofern, als diese die wahrgenommene Preisfairness der Kunden erhöht, ohne eine vollkommene Vergleichbarkeit der Produkte zu ermöglichen.

Zusammenfassend lässt sich aus der Studie von Hermann/Xia/Monroe/Huber (2007) für das Preiscontrolling die zentrale Erkenntnis ableiten, dass der Fokus nicht ausschließlich auf finanziellen Ergebnisgrößen liegen darf, sondern dass vielmehr auch nichtmonetäre Aspekte wie die wahrgenommene Preisfairness $\mathrm{zu}$ integrieren, erfassen und steuern sind, da diese sich mittelfristig auf den durchgesetzten Preis auswirken. Hierfür bedarf es einer intensiven und intelligenten Marktforschung als zentraler Informationsquelle des Marketingcontrollings.

\section{Ausgestaltung von Preispromotionen}

Die Frage nach der optimalen Ausgestaltung von Promotionen beschäftigt sowohl die Praxis als auch die Wissenschaft bereits seit langem. Neben der grundsätzlichen Entscheidung über die Nutzung von Promotionen sind insbesondere Aspekte wie die Dauer oder die Bestimmung der Adressaten für die Promotion von zentraler Bedeutung.

Anderson/Simester (2004) führen in diesem Zusammenhang drei Experimente zur Überprüfung der kurz- und langfristigen Wirkung von temporären Promotionen durch. Dabei manipulieren sie in Kooperation mit einem Versandhaus anhand zweier verschiedener Testkataloge einmalig die Preise eines konstanten Anteils der Katalogartikel. Während einer Kundengruppe (Promotiongruppe) diese Artikel um $60 \%$ reduziert dargeboten werden, erhält eine Kontrollgruppe die gleichen Artikel lediglich um $30 \%$ reduziert. Dabei erfolgt die Manipulation des Katalogs nur einmal: In den folgenden $\mathrm{Pe}$ rioden erhalten alle Probanden wieder den regulären Katalog mit allen Produkten zu herkömmlichen Preisen.

Anderson/Simester (2004) kommen zu den folgenden, für die Praxis relevanten Ergebnissen bei Erstkäufern und bei bestehenden Kunden:

Positiver Kurzfristeffekt bei Erstkäufern und bestehenden Kunden durch

\section{Preispromotion}

Sowohl bei den bestehenden Kunden als auch bei den Erstkunden ist der Anteil derer, welche unmittelbar nach Erhalt des Kataloges eine Bestellung aufgeben, in der Promotiongruppe signifikant höher als in der Kontrollgruppe. Gleichzeitig werden nach Erhalt des Promotionkatalogs signifikant mehr Bestellungen pro Kunde aufgenommen. Der durch die höhere Preisreduktion geringere Stückpreis wird durch die signifikant höhere Bestellmenge in der Promotionengruppe sogar überkompensiert. Folglich kann der kurzfristige Effekt einer Promotion als positiv angesehen werden.

\section{Negativer Langfristeffekt bei \\ bestehenden Kunden durch \\ Preispromotion}

Die Ergebnisse von Anderson/Simester (2004) zeigen allerdings auch, dass eine Fokussierung auf die unmittelbaren Wirkungen temporärer Preisreduktionen zu kurz greift: In der Folgeperiode, in welcher beiden Experimentiergruppen ausschließlich der Katalog mit regulären Preisen zugesandt wird, ist bei den bestehenden Kunden in der Promotiongruppe die Anzahl der Bestellungen signifikant niedriger als bei jenen in der Kontrollgruppe. Auch die Bestellmenge pro Kunde und der Durchschnittspreis pro verkaufter Einheit sind in der Promotiongruppe signifikant niedriger. Dadurch ergibt sich bei den bestehenden Kunden insgesamt ein negativer Effekt der Promotion für das Versandhaus. Anderson/Simester (2004) führen dieses Ergebnis insbesondere auf die erhöhte Preissensibilität derKundenzurück. Für dieUnternehmenspraxis bedeutet dies Folgendes: Das Preiscontrolling sollte berücksichtigen, dass kurzfristige Preispromotionen die Kunden zu Schnäppchenjägern erziehen können und damit verheerende Auswirkungen auf die langfristige Durchsetzung
WWW.GABLER.DE

Shared Services,

\section{BPO und Offshoring}

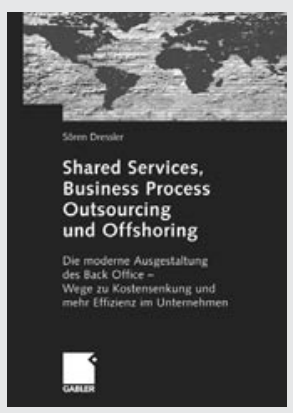

Sören Dressler

Shared Services,
Business Process Out-
sourcing und Offshoring sourcing und Offshoring des Back Office -Wege zu Kostensenkung und mehr Effizienz im Unternehmen

2007. 216 S., Br. EUR 39,90

ISBN 978-3-8349-0257-3

Ein praktischer Leitfaden zur Umsetzung von Shared Services und Business Process Outsourcing sowie zur Wahl der richtigen Standorte. Mit konkreten Handlungsempfehlungen und vielen Best- Practice-Beispielen.

Einfach bestellen: kerstin.kuchta@ gwv-fachverlage.de Telefon +49 (o)611. 7878-626

KOMPETENZ IN SACHEN WIRTSCHAFT

Änderungen vorbehalten Erhältlich im Buchhande oder beim Verlag. 
hoher Preise und somit den Unternehmenserfolg haben können. Diese Erkenntnis steht in Einklang mit weiteren empirischen Studien wie jener von Sun (2005). Im Rahmen dieser Untersuchung konnte nachgewiesen werden, dass kurzfristige Preispromotionen dazu führen, dass Konsumenten nicht nur ihre Kaufentscheidung, sondern auch ihren Konsum vom Aktionszyklus der Unternehmen abhängig machen und sich entsprechend strategisch verhalten (vgl. Sun 2005, S. 430).

\section{Positiver Langfristeffekt bei Erstkäufern durch Preispromotion}

Während, wie oben dargelegt, die langfristigen Auswirkungen von Promotionen bei den bestehenden Kunden negativ sind, konnten Anderson/Simester (2004) in einem zweiten Experiment zeigen, dass bei den Erstkäufern Promotionen langfristig positiv wirken können: Jene Erstkäufer der Promotiongruppe bestellten ebenfalls in der Folgeperiode, wenn auch zu einem geringeren Durchschnittspreis, signifikant mehr Produkte als jene der Kontrollgruppe. Anderson/Simester (2004) führen dieses Ergebnis auf die geringere Erfahrung der Erstkäufer mit dem Angebot des Unternehmens zurück. Ein drittes Experiment zur Kontrolle der Ergebnisse bestätigt diesen Zusammenhang.

Eine Zusammenfassung der experimentellen Untersuchungen von Anderson/Simester (2004) legt nahe, dass Promotionen nur bei Erstkäufern und nicht bei bestehenden Kunden durchgeführt werden sollten. Dass diese Betrachtung allerdings isoliert ist und somit einem Trugschluss unterliegen kann, verdeutlichen die obigen Ausführungen zur wahrgenommenen Preisfairness. Eine Segmentierung zwischen Erstkäufern und bestehenden Kunden hinsichtlich der Promotionplanung ist folglich nur dann sinnvoll, wenn Interaktionen zwischen diesen beiden Segmenten auszuschließen sind. Dies ist aber meistens nicht der Fall.

\section{Delegation von Preiskompetenz an den Außendienst}

Einen weiteren aktuellen und sehr praxisrelevanten Aspekt stellt die Entscheidung über den optimalen Grad der Delegation von Preiskompetenz an den Außendienst dar. Eine Vielzahl konzeptioneller wissenschaftlicher Arbeiten weist auf die Notwendigkeit einer vollkom- menen Delegation von Preisentscheidungen hin. Begründet wird dies mit der Nähe des Außendienstes zum Kunden sowie dessen besseren Kenntnis der verschiedenen Preisbereitschaften im Markt. Andererseits sprechen sich einige Wissenschaftler gegen eine solche Entscheidungsfreiheit des Außendienstes bei der Preisfestlegung aus. Hierbei liegen die Annahmen zugrunde, dass Außendienstmitarbeiter mit hoher Preiskompetenz auch wenn diese mit der Kennzahl Brutto- oder Nettomarge gesteuert werden dazu tendieren, weniger Zeit in die Identifikation von Kunden mit hoher Zahlungsbereitschaft $\mathrm{zu}$ investieren, und eher zu Preiszugeständnissen neigen (Stephenson/Cron/Frazier 1979). Dies führt zu einem niedrigeren durchgesetzten Preis und folglich zu einer geringeren langfristigen Profitabilität. Hierbei zeigt sich insbesondere eine Parallele zu den Überlegungen zur Ausgestaltung von Preispromotionen.

Anhand eines Modells untersucht Joseph (2001) den optimalen Preisgestaltungsspielraum des Außendienstes. Dabei greift er jenen beschriebenen Trade-off zwischen dem Vorteil einer besseren Kenntnis des Marktes und dem Nachteil einer geringeren Anstrengung bei der Identifikation von Kunden mit hoher Zahlungsbereitschaft auf. Joseph (2001) empfiehlt eine geringe Preiskompetenz des Außendienstes, wenn die Suche nach den besonders zahlungsbereiten Kunden mit einem mittleren Schwierigkeitsgrad verbunden ist. Dies ist insofern einleuchtend, als in diesem Fall der Außendienst dazu veranlasst wird, Kunden mit höheren Zahlungsbereitschaften zu identifizieren und die moderaten Suchkosten durch die höheren erzielten Preise überkompensiert werden. Zudem kommt Joseph (2001) zu dem Ergebnis, dass eine hohe Preiskompetenz des Außendienstes dann optimal ist, wenn es sehr schwierig bzw. sehr leicht ist, nicht preissensible Kunden zu identifizieren. Die Gründe hierfür sind allerdings verschieden: Bei einem geringen Schwierigkeitsgrad ist eine Einschränkung der Preiskompetenz des Außendienstes insofern nicht notwendig, als dieser nicht zur Identifikation der besonders zahlungsbereiten Kunden gezwungen werden muss. Bei einem hohen Schwierigkeitsgrad hingegen rechtfertigen die hohen Suchkosten nicht die Identifikation solcher Kunden, weswegen dem Außendienst durch eine hohe Preiskompetenz ein gewisser Spiel- raum bei der Auswahl potenzieller Kunden eingeräumt werden sollte.

Eine Ergänzung der Befunde von Joseph (2001) stellt die Studie von Mishra/Prasad (2004) dar. Hiernach ist eine hohe Preiskompetenz des Außendienstes dann nicht mehr vorteilhaft, wenn es dem Controlling gelingt, die ehemals privaten Informationen des Außendienstes über die Zahlungsbereitschaft im Markt dem Unternehmen und den Vertriebsmanagern zugänglich zu machen. Dies stellt allerdings hohe Anforderungen an das Marketingcontrolling, da Außendienstmitarbeiter solche „privaten" Informationen in der Regel nicht in Systeme integrieren möchten.

Die Ergebnisse der Studien von Joseph (2001) und Mishra/Prasad (2004) haben folgende Implikationen für die Praxis: Bezüglich der Festlegung der Preiskompetenz des Außendienstes hat der Vertriebsmanager vor allem zwei Aspekte zu berücksichtigen: Erstens das umfangreichere Wissen des Außendienstes über die Zahlungsbereitschaften im Markt sowie zweitens ein eventuelles dysfunktionales Verhalten des Außendienstmitarbeiters, welches sich insbesondere in einer geringeren Anstrengung bei der Identifikation nicht preissensitiver Kunden widerspiegelt. Während ein mögliches dysfunktionales Verhalten des Außendienstes per se eine Beschränkung der Preiskompetenz nahe legt, spricht die Nähe des Außendienstes zum Markt für eine hohe Preiskompetenz desselbigen. Wie die Studie von Mishra/Prasad (2004) zeigt, kann die Nähe des Außendienstes zum Markt allerdings auch dann ausgeschöpft werden, wenn es dem Marketingcontrolling gelingt, die privaten Informationen des Außendienstes dem Unternehmen zugänglich zu machen. Dies stellt insbesondere bei einer Einschränkung der Preiskompetenz des Außendienstes eine lohnenswerte Herausforderung dar, da in diesem Fall der Vorteil der besseren Marktkenntnis des Außendienstes weiterhin gegeben ist und gleichzeitig ein mögliches dysfunktionales Verhalten des Außendienstes begrenzt werden kann.

\section{Literatur}

1. Reinecke, S./Janz, S. (2007): Marketingcontrolling, Stuttgart 2007.

2. Stephenson, R. P./Cron, W. L./Frazier, G. L. (1979): Delegating Pricing Authority to the Salesforce: The Effects of Sales and Profit Performance, in: Journal of Marketing, 43, 1, $21-28$. 


\title{
Bachelor-Lehrbuch
}

\section{zum Management}



Georg Schreyögg | Jochen Koch

Grundlagen des Managements

Basiswissen für Studium und Praxis

2007. XIV, $461 \mathrm{~S}$.

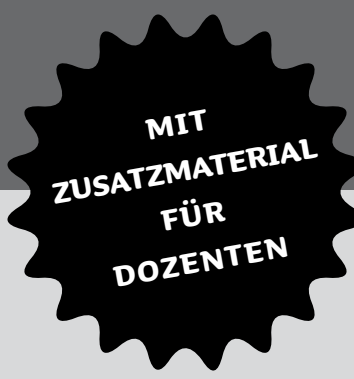

Br. EUR 24,90 ISBN 978-3-8349-0376-1

Das neue Lehrbuch von Georg Schreyögg und Jochen Koch gibt eine kompakte Darstellung der wichtigsten Inhalte des Managements. Themenauswahl und -aufbereitung sind speziell auf die Anforderungen an Studierende in Bachelor-Studiengängen zugeschnitten und das gesamte Lehrbuch ist auf ein Standardmodul in der Bachelor-Ausbildung hin konzipiert. Die einzelnen Lerneinheiten folgen dabei einem einheitlichen didaktischen Konzept: Jedes Kapitel enthält Lernziele und Schlüsselbegriffe, einen geschlossenen Lehrtext mit integrierten Informationskästen und Marginalienkommentierung, Lern- und Diskussionsfragen sowie eine abschließende Fallstudie zu Übungszwecken. Zusätzliche Service-Komponenten sollen den Einsatz als Basislehrbuch erleichtern: Alle Leserinnen und Leser können Lösungshinweise zu den Lernkontrollfragen von der Verlags-Homepage unter www.gabler.de herunterladen. Für Dozentinnen und Dozenten sind dort außerdem Zusatzmaterialien zur Unterrichtsvorbereitung hinterlegt, insbesondere Lösungshinweise für die Diskussionsfragen, Musterlösungen für die Fallstudien sowie fertige Foliensätze zur Präsentation der Lehrinhalte.

\section{Die Autoren}

Prof. Dr. Georg Schreyögg und Dr. Jochen Koch forschen und lehren am Institut für Management, Freie Universität Berlin.

\author{
Aus dem Inhalt \\ - Management: Einführung und konzeptionelle Grundlagen \\ _ Planung und Kontrolle \\ _ Organisation, Führung und Personaleinsatz
}

